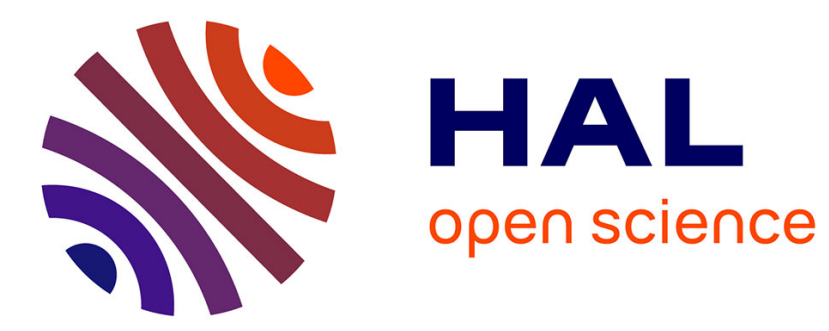

\title{
Fast domain wall motion governed by topology and OErsted fields in cylindrical magnetic nanowires
}

M. Schöbitz, A. de Riz, S. Martin, S. Bochmann, C. Thirion, J. Vogel, M. Foerster, L. Aballe, T. O. O Menteş, A. Locatelli, et al.

\section{- To cite this version:}

M. Schöbitz, A. de Riz, S. Martin, S. Bochmann, C. Thirion, et al.. Fast domain wall motion governed by topology and OErsted fields in cylindrical magnetic nanowires. Physical Review Letters, 2019, 123 (21), pp.217201. 10.1103/PhysRevLett.123.217201 . hal-02075418

\section{HAL Id: hal-02075418 \\ https://hal.science/hal-02075418}

Submitted on 20 Dec 2019

HAL is a multi-disciplinary open access archive for the deposit and dissemination of scientific research documents, whether they are published or not. The documents may come from teaching and research institutions in France or abroad, or from public or private research centers.
L'archive ouverte pluridisciplinaire HAL, est destinée au dépôt et à la diffusion de documents scientifiques de niveau recherche, publiés ou non, émanant des établissements d'enseignement et de recherche français ou étrangers, des laboratoires publics ou privés. 


\title{
Fast Domain Wall Motion Governed by Topology and Ersted Fields in Cylindrical Magnetic Nanowires
}

\author{
M. Schöbitz, ${ }^{1,2,3,{ }^{*}}$ A. De Riz, ${ }^{1}$ S. Martin, ${ }^{1,3}$ S. Bochmann, ${ }^{2}$ C. Thirion, ${ }^{3}$ J. Vogel $\odot,{ }^{3}$ M. Foerster $\odot,{ }^{4}$ L. Aballe, ${ }^{4}$ \\ T. O. Menteş๑, ${ }^{5}$ A. Locatelli®, ${ }^{5}$ F. Genuzio $\odot,{ }^{5}$ S. Le-Denmat, ${ }^{3}$ L. Cagnon, ${ }^{3}$ J. C. Toussaint, ${ }^{3}$ D. Gusakova, ${ }^{1}$ \\ J. Bachmann $\oplus^{2,6}$ and O. Fruchart $\oplus^{1, \dagger}$ \\ ${ }^{1}$ Univ. Grenoble Alpes, CNRS, CEA, Spintec, 38054 Grenoble, France \\ ${ }^{2}$ Friedrich-Alexander-Universität Erlangen-Nürnberg, Inorganic Chemistry, 91058 Erlangen, Germany \\ ${ }^{3}$ Univ. Grenoble Alpes, CNRS, Institut Néel, 38042 Grenoble, France \\ ${ }^{4}$ Alba Synchrotron Light Facility, CELLS, 08290 Barcelona, Spain \\ ${ }^{5}$ Elettra-Sincrotrone Trieste S.C.p.A., Basovizza, 34149 Trieste, Italy \\ ${ }^{6}$ Institute of Chemistry, Saint Petersburg State University, St. Petersburg 198504, Russia
}

(Received 9 July 2019; published 21 November 2019)

\begin{abstract}
While the usual approach to tailor the behavior of condensed matter and nanosized systems is the choice of material or finite-size or interfacial effects, topology alone may be the key. In the context of the motion of magnetic domain walls (DWs), known to suffer from dynamic instabilities with low mobilities, we report unprecedented velocities $>600 \mathrm{~m} / \mathrm{s}$ for DWs driven by spin-transfer torques in cylindrical nanowires made of a standard ferromagnetic material. The reason is the robust stabilization of a DW type with a specific topology by the CErsted field associated with the current. This opens the route to the realization of predicted new physics, such as the strong coupling of DWs with spin waves above $>600 \mathrm{~m} / \mathrm{s}$.
\end{abstract}

DOI: 10.1103/PhysRevLett.123.217201

It is well known that specific properties in condensedmatter and nanosized systems can be obtained by either acting on the electronic structure by selecting an appropriate material composition and crystalline structure, or by making use of finite-size and interfacial effects, strain, gating with an electric field, etc. [1]. These approaches have proven suitable for tailoring charge transport, optical properties, electric or magnetic polarization, etc., however, there are limits regarding what can be achieved with materials, or realized with device fabrication. An alternative strategy entails considering a specific topology in order to develop the desired properties of a system, yielding diverse applications such as the design of wide-band-gap photonic crystals [2] and the control of flow of macromolecules [3], or novel theoretical methods such as for the description of defects [4], or intringuing 3D vector-field textures such as hopfions and torons [5]. As regards magnetism, unusual properties resulting from topological features have been predicted, such as the existence of a domain wall (DW) in the ground state of a Moebius ring [6], or the nonreciprocity of spin waves induced by curvature and boundary conditions in nanotubes [7].

Here, we show that topology plays a critical role in the physics of DW motion in one-dimensional conduits, a prototypical case for magnetization dynamics. For the sake of simplicity of fabrication and monitoring, DW motion under magnetic field or spin-polarized current is usually conducted in planar systems, made of stacked thin films patterned laterally by lithography. In them, DWs are dynamically unstable above a given threshold of field or current (Walker limit), undergoing transformations of their magnetization texture associated with a drastic drop in their mobility. Ways are being investigated to overcome this limitation through the engineering of microscopic properties. Two major routes are the use of the Dzyaloshinskii-Moriya interaction in order to stabilize the walls [8-10], or of natural or synthetic ferrimagnets with vanishing magnetization to decrease the angular momentum in order to increase spin-transfer torque efficiency and boost the precessional frequency [11-13].

The three-dimensional nature of cylindrical nanowires (NWs) gives rise to the existence of a DW with a specific topology, which respects the rotational invariance and circular boundary conditions. It is named the Bloch-point wall (BPW) [14] and has been experimentally confirmed only recently $[15,16]$. It was predicted that this wall can circumvent the Walker limit, but field-driven motion experiments disappointingly failed to confirm a topological protection [17]. Here, we report experimental results on current-induced DW motion in such NWs. We show that although previously disregarded, the Ersted field induced by the current plays instead a crucial and valuable role in stabilizing BPWs, contrary to the field-driven case. This allows them to retain their specific topology and thus reach velocities $>600 \mathrm{~m} / \mathrm{s}$ in the absence of Walker breakdown, which is quantitatively consistent with predictions.

DWs with two distinct topologies exist in NWs: the transverse-vortex wall (TVW) and the BPW 
[Figs. 1(a), 1(b)]. The former has the same topology as all DW types known in 2D flat strips [18]. The latter is found only in NWs and exhibits azimuthal curling of magnetic moments around a Bloch point, a local vanishing of magnetization $[19,20]$. This unique topological feature of NWs is at the origin of the predicted fast speed and stability during magnetic-field or current-driven motion of BPWs. This is easily explained by considering the time derivative of the magnetization vector $\dot{\mathbf{m}}$ at any point, described by the Landau-Lifshitz-Gilbert equation [21].

$\dot{\mathbf{m}}=-\gamma_{0} \mathbf{m} \times \mathbf{H}+\alpha \mathbf{m} \times \dot{\mathbf{m}}-(\mathbf{u} \cdot \boldsymbol{\nabla}) \mathbf{m}+\beta \mathbf{m} \times[(\mathbf{u} \cdot \nabla) \mathbf{m}]$,

with $\gamma_{0}=\mu_{0}|\gamma|, \gamma$ being the gyromagnetic ratio, $\alpha \ll 1$ the Gilbert damping parameter, and $\beta$ the nonadiabaticity parameter. $\mathbf{H}$, the total effective field, is comprised of applied fields and fields originating from magnetic anisotropy, exchange, and dipolar energy. The spin-polarized part of the charge current induces so-called spin-transfer torques, taken into account through $\mathbf{u}$, with $|\mathbf{u}|=P\left(j \mu_{B} / e M_{s}\right)$ [21]. $j$ and $P$ are the charge current and its spin-polarization ratio, respectively, $\mu_{B}$ is the Bohr magneton, $e$ the elementary charge, and $M_{s}$ the spontaneous magnetization.

In purely field driven cases, the applied field favors the precession of $\mathbf{m}$ around the field direction. In flat strips, for applied fields above a few $\mathrm{mT}$ this causes repeated DW transformations from transverse to vortex walls for in-plane magnetization, and from Néel to Bloch walls for out-ofplane magnetization. This so-called Walker breakdown [22] is facilitated by the fact that all these DW configurations share the same topology [23-25]. The mobility is high below the Walker threshold field (scaling with $1 / \alpha$ ) and low above (scaling with $\alpha$ ). The same physics is expected in NWs for the TVW, with the Walker field equal to zero due to the rotational symmetry $[7,14]$. The phenomenology of currentdriven cases is similar: the adiabatic term favors motion, the nonadiabatic term favors azimuthal precession [third and fourth terms in Eq. (1), respectively], and the DW velocity is expected to be $\approx(\beta / \alpha) u$ below the Walker threshold and $\approx u$ above it $[21,24]$, again with a vanishing threshold for TVWs in NWs [26].

In contrast to these cases, one expects that magnetization cannot freely precess azimuthally in a BPW, since it would periodically imply a head-on or tail-on configuration along all three axes around the Bloch point, with an enormous cost in dipolar energy. Instead, the azimuthal rotation should come to a halt and remain in a state essentially similar to the static one [Fig. 1(b)]. This implies an absence of Walker breakdown, both under field $[7,14]$ and current $[27,28]$, and steady-state motion of the wall. The steady circulation is expected to be clockwise $(\mathrm{CW})$ with respect to the direction of motion of the DW, while the counterclockwise (CCW) circulation may undergo a dynamicsinduced irreversible switching event to recover the $\mathrm{CW}$ (a)

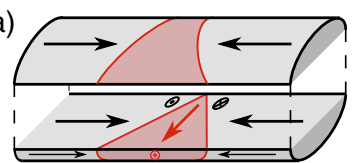

(b)

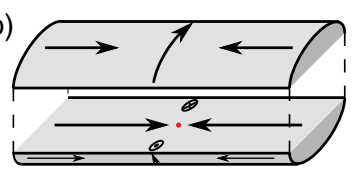

(c)

XMCD-PEEM image

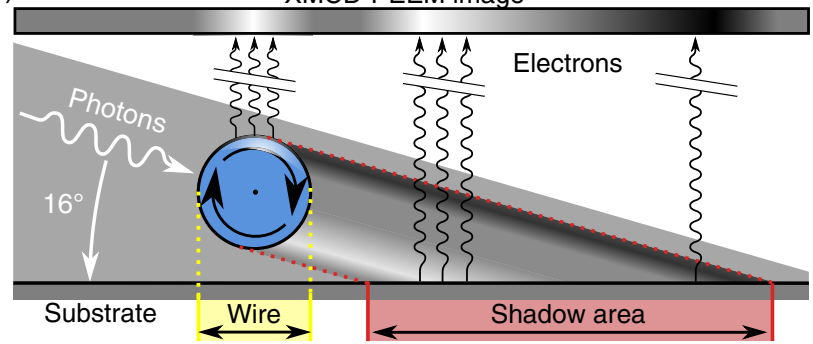

FIG. 1. Schematic of (a) a TDW and (b) a BPW. (c) Schematic of shadow XMCD-PEEM and the contrast resulting from a BPW.

circulation and steady state. This picture is valid both for BPWs in wires [14,27], and vortex walls [7,28] in thickwalled tubes. Thanks to this locked topology, the mobility of the BPW is expected to remain high under both field and current. Only when a speed around $\approx 1000 \mathrm{~m} / \mathrm{s}$ is attained, the speed is predicted to reach a plateau, with new physics expected to occur via interactions with spin waves, known as the spin-Cherenkov effect [7]. However, so far there exists no experimental report of the mobility of any of these walls under neither magnetic field nor current.

Our Letter is based on magnetically soft $\mathrm{Co}_{30} \mathrm{Ni}_{70}$ wires with diameter $90 \mathrm{~nm}$, electroplated in anodized alumina templates [29]. Following the dissolution of the latter, isolated wires lying on a $\mathrm{Si}$ substrate are contacted with pads to allow for the injection of electric current. DWs were monitored with both magnetic force microscopy (MFM) and $\mathrm{x}$-ray magnetic circular dichroism photo-emission electron microscopy (XMCD-PEEM) in the shadow mode [Fig. 1(c)] to reveal the three-dimensional texture of magnetization [16,30,31]. While in MFM, sharp ns-long pulses could be sent, in XMCD-PEEM the shape of current pulses was distorted to a minimum width of 10-15 ns, due to long cabling, UHV feedthroughs and the sample holder contacts. Micromagnetic simulations were carried out with the homemade finite-element code FeeLLGood [32], based on the Landau-Lifshitz-Gilbert equation including spin-transfer torques. See Supplemental Material for additional details on the methods [33].

Domain wall velocities were experimentally investigated primarily with MFM imaging. Figure 2(b) shows an atomic force microscopy (AFM) image of the left-hand side of the contacted NW from Fig. 2(a). The corresponding MFM image in Fig. 2(c) shows the initial magnetic configuration, with two DWs located at 1.2 and $7.2 \mu \mathrm{m}$ from the edge of the left contact. By applying a current pulse of duration $5.8 \mathrm{~ns}$ and amplitude $2.2 \times 10^{12} \mathrm{~A} / \mathrm{m}^{2}$, the left hand DW moved over a distance of $\approx 2 \mu \mathrm{m}$ [Fig. 2(d)], corresponding to an average velocity of $\approx 350 \mathrm{~m} / \mathrm{s}$. However, the right-hand 
DW remains pinned, highlighting a common and key issue for inferring DW velocities from motion distances: pinning on geometrical or microstructural defects hampers DW motion [44]. Depinning not only requires a current density above a critical value $j_{\mathrm{dp}}$, but repinning can also occur at another location with a deeper energy well, while the current pulse is still being applied. This results in DW propagation with an effective time span possibly much shorter than the nominal pulse duration. Consequently, the values for DW velocity converted from motion distance and nominal pulse length are a lower bound of an unknown higher velocity (see Supplemental Material [33] for a quantitative discussion). Furthermore, with such large current densities the effect of Joule heating may not be neglected. However, measurements of the NW resistance during the pulse showed that the samples never exceeded the Curie temperature (see Supplemental Material [33]) and that the results described herein are not caused by thermal activation.

Figure 2(e) (open circles) shows the discussed lower bound for DW velocity, as a function of applied current density, inferred from a multitude of MFM images before and after pulses with durations ranging from 5 to $15 \mathrm{~ns}$. Consistent with the expected occurrence of re-pinning, lower velocities are inferred from longer pulse durations.

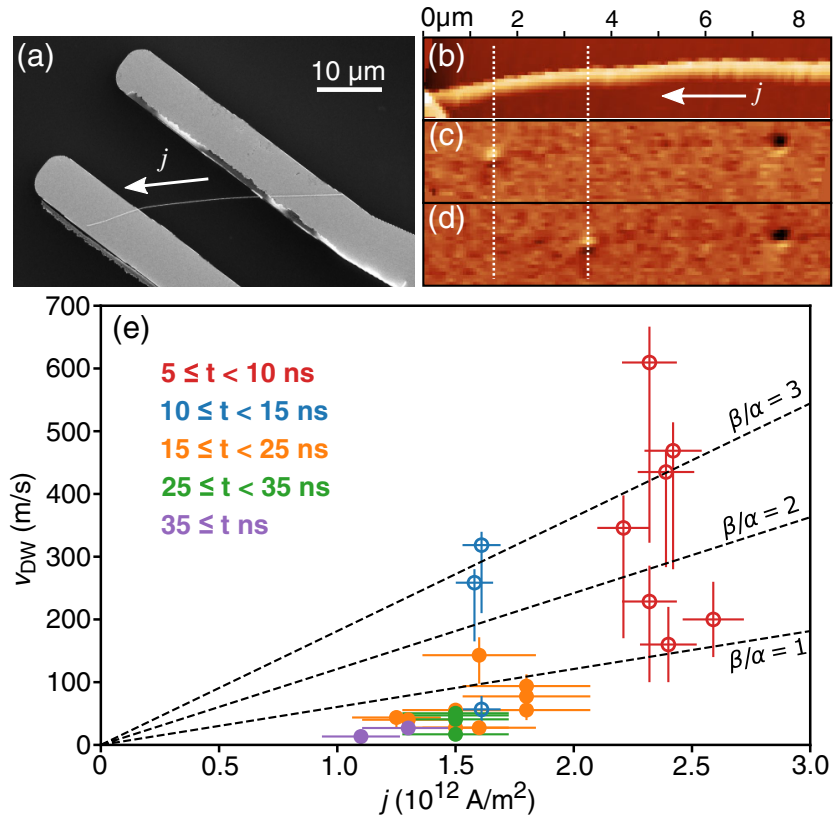

FIG. 2. (a) SEM, (b) AFM, and corresponding (c),(d) MFM images of a $90 \mathrm{~nm}$ diameter $\mathrm{Co}_{30} \mathrm{Ni}_{70} \mathrm{NW}$ with $\mathrm{Ti} / \mathrm{Au}$ electrical contacts. (c) Initial state, with two DWs. (d) Same wire, after a current pulse with $2.2 \times 10^{12} \mathrm{~A} / \mathrm{m}^{2}$ magnitude and $5.8 \mathrm{~ns}$ duration. (e) Domain wall velocity as a function of applied current density and duration (see inner caption), monitored with MFM (open circles) and XMCD PEEM (filled circles) from four individual NWs. The dashed lines are expectations from the onedimensional model below the Walker breakdown, for $v=(\beta / \alpha) u$ with $\beta / \alpha=1,2,3$.
Still, DW velocities up to $>600 \mathrm{~m} / \mathrm{s}$ were observed for applied current densities $\approx 2.4 \times 10^{12} \mathrm{~A} / \mathrm{m}^{2}$. This sets a fivefold record for purely spin-transfer torque motion of DWs in a standard ferromagnetic material, i.e., with large magnetization, with reported values hardly exceeding $100 \mathrm{~m} / \mathrm{s}$ [45]. Similar or higher speeds have been measured recently, however, in low-magnetization ferrimagnets, thereby enhancing the efficiency of spin-transfer torque [46]. Here, it is the topology of the wall that enhances the DW speed, not a special material. Similarly, these DW velocity measurements are not distorted by DW inertia, since simulations showed that this effect will only come into play in subnanosecond pulse experiments (see Supplemental Material [33]). The black dotted lines in Fig. 2(e) act as a guide to the eye through the speed predicted by the one-dimensional model below the Walker breakdown $v=(\beta / \alpha) u$, for three different ratios of $\beta / \alpha: 1,2$, and 3 (for $\mathrm{Co}_{30} \mathrm{Ni}_{70} M_{s}=0.67 \mathrm{MA} / \mathrm{m}^{2}$, $P \approx 0.7$, resulting in $u \approx 60.4 \mathrm{~m} / \mathrm{s}$ per $10^{12} \mathrm{~A} / \mathrm{m}^{2}$ ). This is not intended as precise modeling, but rather to show that the experiments are clearly not compatible with $v=u$, supporting the absence of Walker breakdown for the BPW. Instead a value of $\beta / \alpha \gtrsim 3$ is inferred. Note, however, that the adverse effects of DW pinning reappear in the form of a threshold current density $j_{\mathrm{dp}} \approx 1.2 \times 10^{12} \mathrm{~A} / \mathrm{m}^{2}$ required to set any DW in motion. Even above this value, DW motion was not fully reproducible, with some pinning sites associated with a larger $j_{\mathrm{dp}}$.

To link unambiguously the measured velocity with theory, the DW type must be identified. For this purpose, we employed shadow XMCD-PEEM and imaged NWs before and after injecting a given current pulse [Figs. 3(a), 3(b), and full symbols in Fig. 2(e)]. Note that the values for speed are lower than those measured with MFM, as expected for less sharp pulse shapes with consequentially larger width. Returning to the DW type, the first striking fact is the following: from hundreds of DWs imaged after current injection, all were of the BPW type. These unambiguously appear as a symmetric bipolar contrast in the shadow [16], corresponding to azimuthal rotation of magnetization as on Figs. 3(a)-3(b). This sharply contrasts with all our previous observations of NWs, imaged in the as-prepared state or following a pulse of magnetic field, for which both TVWs and BPWs had been found in sizable amounts $[16,17]$. The second striking fact is that the sign of the BPW circulation is deterministically linked to the sign of the latest current pulse, provided that its magnitude is above a rather well-defined threshold which, as shown in Fig. 3(c), lies around $1.4 \times 10^{12} \mathrm{~A} / \mathrm{m}^{2}$. In contrast with a one-time Walker event discussed previously, this holds true irrespective of whether or not the wall has moved under the stimulus of the current pulse, and is independent of the pulse duration at the probed timescales. We hypothesize that these two facts are related to the Ersted field associated with the longitudinal electric current, its azimuthal direction favoring the BPW with a 
(a)

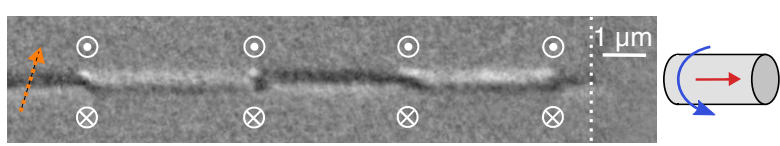

(b)

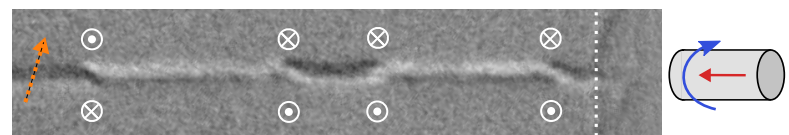

(c)

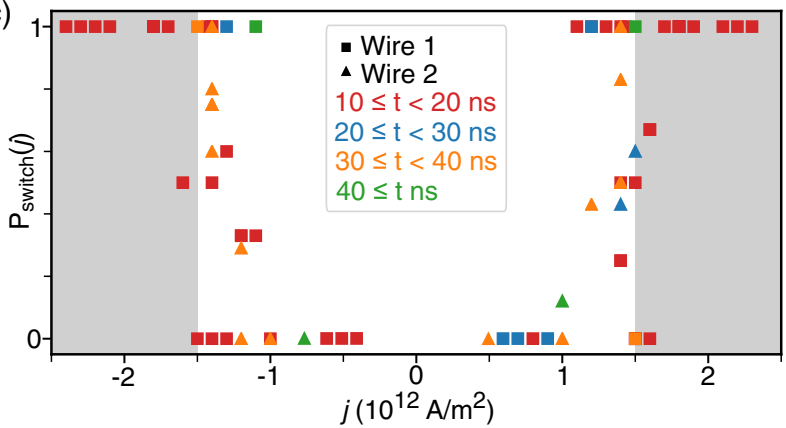

FIG. 3. (a), (b) Consecutive XMCD-PEEM images of a NW with a tilted $\mathrm{X}$-ray beam (orange arrow). The azimuthal circulation of the four BPWs seen in the NW shadow is indicated by the white arrows, consistent with the Ersted field of the previously applied current (blue and red arrows in the right-hand schematic, respectively). From (a) to (b), a $15 \mathrm{~ns}$ and $1.4 \times 10^{12} \mathrm{~A} / \mathrm{m}^{2}$ current pulse switches $75 \%$ of BPWs. DW displacement from (a) to (b) cannot be discussed as directly resulting from spin-transfer torque, and the density of current lies below the threshold for free motion (c) BPW switching probability as a function of $j$ for two different wire samples (squares and triangles). Pulse durations are categorized and color coded, see included labels. The gray region indicates the current density required for switching in simulations.

given circulation. Indeed, for a uniform current density $j$, the Ersted field is $H=j r / 2$ at distance $r$ from the NW axis. For the present NWs with radius $R=45 \mathrm{~nm}$ and $j=$ $1 \times 10^{12} \mathrm{~A} / \mathrm{m}^{2}$ this translates to $28 \mathrm{mT}$ at the NW surface, which is a significant value.

In order to support this claim, we conducted micromagnetic simulations including the Ersted field, which had not been considered in previous works. Starting from a DW at rest with $R=45 \mathrm{~nm}$, we used $\alpha=1$ to avoid ringing effects and obtain a quasistatic picture, suitable to describe the PEEM experiments, for which the pulse rise time is several nanoseconds. We evidenced that while the added effect of spin-transfer torques may alter the transformation mechanisms, it is of minor importance compared to the Ersted field and considering or disregarding these torques does not quantitatively impact switching. Accordingly, below we present only results disregarding these torques. With an applied Ersted field, within the domains the peripheral magnetization tends to curl around the axis, while it remains longitudinal on the NW axis. We first consider TVWs as the initial state and find that these transform into BPWs with $\mathrm{CW}$ circulations with respect to the current direction, if the current density exceeds $0.4 \times 10^{12} \mathrm{~A} / \mathrm{m}^{2}$. The underlying process is illustrated on Fig. 4(a), displaying maps of the radial and azimuthal magnetization components, $m_{r}$ and $m_{\varphi}$, respectively, on the unrolled surface of a NW as a function of time. These highlight the locations of the inward and outward flux of magnetization through the surface, signature of a TVW [18]. While these local configurations are initially diametrically opposite, they approach each other until they eventually merge, expelling the transverse core of the wall from the NW. This is associated with the nucleation of a Bloch point at the NW surface, which later on drifts towards the NW axis, ending up in a BPW. This process is similar to the dynamical transformation of a TVW into a BPW upon motion under a longitudinal magnetic field [17], and explains the absence of TVWs in our measurements, for which the applied current densities were always larger than $0.4 \times 10^{12} \mathrm{~A} / \mathrm{m}^{2}$. In order to understand the unique circulation observed, we now consider a BPW as the initial state. BPWs with a circulation matching that of the Ersted field do not change qualitatively, only their width increases during the pulse. On the contrary, BPWs shrink if their initial circulation is CCW, i.e., opposite to the Ersted field. For $j \leq 1.5 \times 10^{12} \mathrm{~A} / \mathrm{m}^{2}$ the CCW BPW reaches a narrow yet stable state, and recovers its initial state after the pulse. Beyond this value the circulation switches through a transient radial orientation of magnetization [Fig. 4(b)]. After the switching of circulation, the BPW expands and reaches a stable $\mathrm{CW}$ state. The value of the critical current density required for circulation switching is in quantitative agreement with the experimental one [Fig. 3(c), $\approx 1.4 \times 10^{12} \mathrm{~A} / \mathrm{m}^{2}$ ], although the simulation does not incorporate thermal activation and considers $\alpha=1$. This suggests that the switching process is robust and intrinsic, in agreement with the narrow experimental distribution of critical current. In our simulations the time required for switching is $<10 \mathrm{~ns}$, though switching times an order of magnitude faster are expected for realistic values of $\alpha<0.1$, which explains why no dependence on the pulse width was observed in the experiments, where all pulse widths were above 5 ns.

In experiments where the DW type was visible, DW motion events were observed for applied current densities larger than the critical current density required for the circulation switching event. Thus, in these the circulation is always $\mathrm{CCW}$ with respect to the propagation direction, i.e., $\mathrm{CW}$ with respect to the current direction, because the charge of electrons is negative. Remarkably, this sense of circulation is opposite to the situation expected when neglecting the Ersted field, which would select the CW circulation with respect to the propagation direction, as dictated by the chirality of the LLG equation $[14,27,28]$. There must therefore be a competition for the circulation sense and for the case of $90 \mathrm{~nm}$ diameter NWs, the CErsted field dominates. Despite this, we find in simulations that the BPW motion still follows $v \approx(\beta / \alpha) u$ whether or not the Ersted field is considered. Notice that the $\beta$ parameter is 

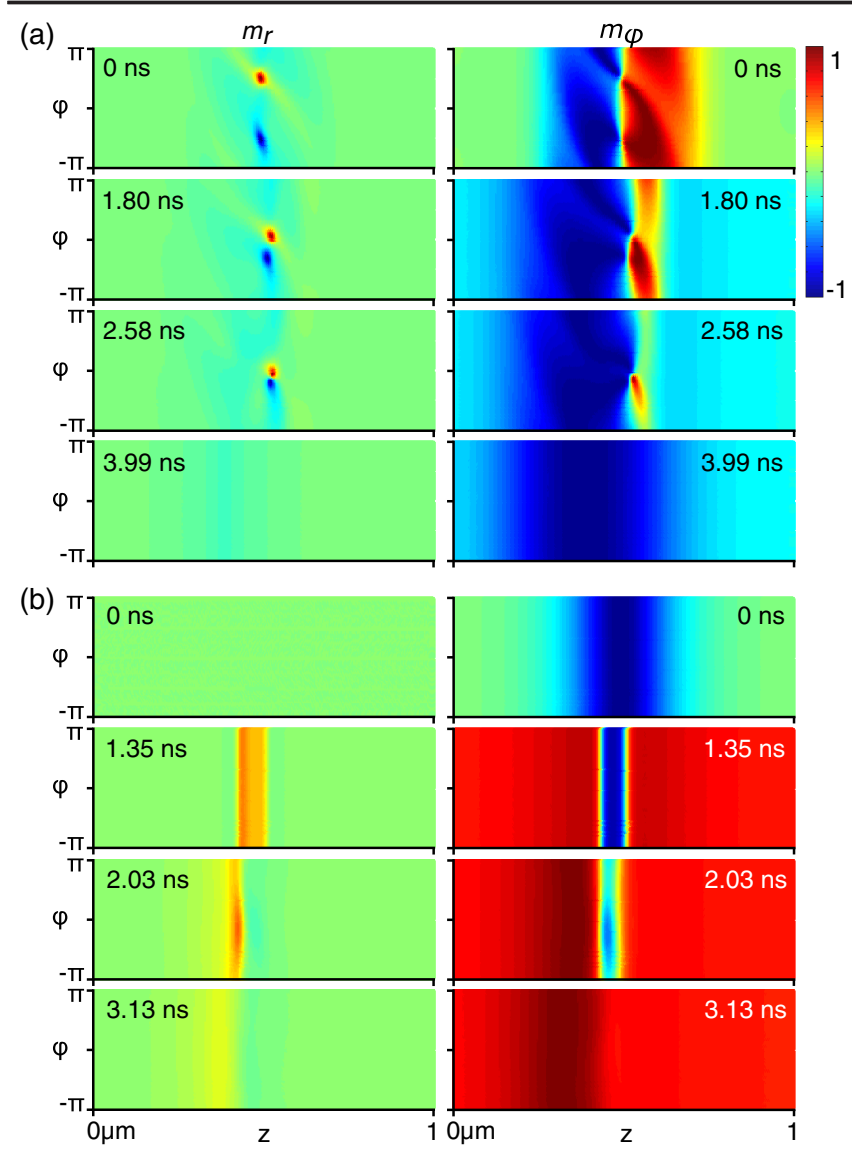

FIG. 4. DW transformations by the Ersted field in micromagnetic simulations for (a) TVW to BPW, with $j=0.4 \times 10^{12} \mathrm{~A} / \mathrm{m}^{2}$, and (b) BPW circulation reversal, with $j=-1.8 \times 10^{12} \mathrm{~A} / \mathrm{m}^{2}$. Left and right are color maps of the radial and azimuthal magnetization components, $m_{r}$ and $m_{\varphi}$, respectively, over time on the unrolled surface of a $90 \mathrm{~nm}$ diameter, $1 \mu \mathrm{m}$-long NW with $\alpha=1$.

expected to depend on the DW width, however, for widths much smaller than the ones studied here [47]. The predictions of high mobility and possibly spinCherenkov effect are thus probably not put into question.

Surprisingly, the Ersted field was previously only considered in a single report for NWs of square cross section [48]. No qualitative impact was found, likely because a NW side of at most $48 \mathrm{~nm}$ was considered, and a simple analytical model describing magnetization in the domain and balancing Zeeman Ersted energy with exchange energy shows that the impact of the Ersted field scales very rapidly as $R^{3}$, a tendency confirmed by simulations. The situation closest to the present case is the report of flat strips made of spin-valve asymmetric stacks [49]. Such strips can be viewed as the unrolled surface of a wire, the curling of the BPW translating into a transverse wall, which tends to be stabilized during motion due to the Ersted field.

To conclude, we have shown experimentally and by simulation that the Ersted field generated by the

spin-polarized current flowing through a cylindrical NW has a crucial impact on DW dynamics, while it had been disregarded so far. This Ersted field robustly stabilizes BPWs, in contrast with the field-driven case [17]. This stabilization allows for the key features predicted for their specific topology to apply $[14,27,28]$ : we evidenced DW velocities in excess of $600 \mathrm{~m} / \mathrm{s}$ confirming the absence of Walker breakdown $[7,50]$ and setting a fivefold record for spin-transfer-torque-driven DW motion in large magnetization ferromagnets [45]. This suggests that the experimental realization of further novel physics is at hand, such as the predicted spin-Cherenkov effect with strong coupling of DWs with spin waves.

M.S. acknowledges a grant from the Laboratoire d'excellence LANEF in Grenoble (ANR-10-LABX-51-01). The project received financial support from the French National Research Agency (Grant No. JCJC MATEMAC3D). This work was partly supported by the French RENATECH network, and by the Nanofab platform (Institut Néel), whose team is greatly acknowledged for technical support. We thank Jordi Prat for his technical support at the ALBA Circe beam line and Olivier Boulle for useful discussions.

"Corresponding author. michael.schobitz@cea.fr Corresponding author. olivier.fruchart@cea.fr

[1] R. E. Newnham, Properties of Materials-Anisotropy, Symmetry, Structure (Oxford University Press, Oxford, 2005).

[2] Y. Lu, Y. Yang, J. K. Guest, and A. Srivastava, Sci. Rep. 7, 43407 (2017).

[3] J. Qin and S. T. Milner, Macromolecules 47, 6077 (2014).

[4] N. D. Mermin, Rev. Mod. Phys. 51, 591 (1979).

[5] P. J. Ackerman and I. I. Smalyukh, Phys. Rev. X 7, 011006 (2017).

[6] O. V. Pylypovskyi, V.P. Kravchuk, D. D. Sheka, D. Makarov, O. G. Schmidt, and Y. Gaididei, Phys. Rev. Lett. 114, 197204 (2015).

[7] M. Yan, C. Andreas, A. Kakay, F. Garcia-Sanchez, and R. Hertel, Appl. Phys. Lett. 99, 122505 (2011).

[8] I. M. Miron, T. Moore, H. Szambolics, L. D. BudaPrejbeanu, S. Auffret, B. Rodmacq, S. Pizzini, J. Vogel, M. Bonfim, A. Schuhl, and G. Gaudin, Nat. Mater. 10, 419 (2011).

[9] A. Thiaville, S. Rohart, E. Jué, V. Cros, and A. Fert, Europhys. Lett. 100, 57002 (2012).

[10] K.-S. Ryu, L. Thomas, S.-H. Yang, and S. Parkin, Nat. Nanotechnol. 8, 527 (2013).

[11] K.-J. Kim, S. K. Kim, Y. Hirata, S.-H. Oh, T. Tono, D.-H. Kim, T. Okuno, W. S. Ham, S. Kim, G. Go, Y. Tserkovnyak, A. Tsukamoto, T. Moriyama, K.-J. Lee, and T. Ono, Nat. Mater. 16, 1187 (2017).

[12] L. Caretta, M. Mann, F. Büttner, K. Ueda, B. Pfau, C. M. Günther, P. Hessing, A. Churikova, C. Klose, M. Schneider, 
D. Engel, C. Marcus, D. Bono, K. Bagschik, S. Eisebitt, and G. S. D. Beach, Nat. Nanotechnol. 13, 1154 (2018).

[13] S.-H. Yang, K.-S. Ryu, and S. Parkin, Nat. Nanotechnol. 10, 221 (2015).

[14] A. Thiaville and Y. Nakatani, in Spin Dynamics in Confined Magnetic Structures III, Domain-wall dynamics in nanowires and nanostrips, (Springer, Berlin, 2006), pp. 161-205.

[15] N. Biziere, C. Gatel, R. Lassalle-Balier, M. C. Clochard, J. E. Wegrowe, and E. Snoeck, Nano Lett. 13, 2053 (2013).

[16] S. Da Col, S. Jamet, N. Rougemaille, A. Locatelli, T. O. Menteş, B. S. Burgos, R. Afid, M. Darques, L. Cagnon, J. C. Toussaint, and O. Fruchart, Phys. Rev. B 89, 180405(R) (2014).

[17] A. Wartelle, B. Trapp, M. Staňo, C. Thirion, S. Bochmann, J. Bachmann, M. Foerster, L. Aballe, T. O. Menteş, A. Locatelli, A. Sala, L. Cagnon, J. C. Toussaint, and O. Fruchart, Phys. Rev. B 99, 024433 (2019).

[18] S. Jamet, N. Rougemaille, J. C. Toussaint, and O. Fruchart, Head-to-head domain walls in one-dimensional nanostructures: An extended phase diagram ranging from strips to cylindrical wires, in Magnetic Nano- and Microwires: Design, Synthesis, Properties and Applications (Woodhead, Cambridge, 2015), pp. 783-811.

[19] R. Feldtkeller, Z. Angew. Phys. 19, 530 (1965).

[20] W. Döring, J. Appl. Phys. 39, 1006 (1968).

[21] A. Thiaville, Y. Nakatani, J. Miltat, and Y. Suzuki, Europhys. Lett. 69, 990 (2005).

[22] N. L. Schryer and L. R. Walker, J. Appl. Phys. 45, 5406 (1974).

[23] G. S. D. Beach, C. Nistor, C. Knuston, M. Tsoi, and J. L. Erskine, Nat. Mater. 4, 741 (2005).

[24] A. Mougin, M. Cormier, J. P. Adam, P. J. Metaxas, and J. Ferré, Europhys. Lett. 78, 57007 (2007).

[25] M. Hayashi, L. Thomas, C. Rettner, R. Moriya, and S. S. P. Parkin, Nat. Phys. 3, 21 (2007).

[26] M. Yan, A. Kákay, S. Gliga, and R. Hertel, Phys. Rev. Lett. 104, 057201 (2010).

[27] R. Wieser, E. Y. Vedmedenko, P. Weinberger, and R. Wiesendanger, Phys. Rev. B 82, 144430 (2010).

[28] J. A. Otalora, J. A. Lopez-Lopez, A. S. Nunez, and P. Landeros, J. Phys. Condens. Matter 24, 436007 (2012).

[29] S. Bochmann, A. Fernandez-Pacheco, M. Mačković, A. Neff, K. R. Siefermann, E. Spiecker, R. P. Cowburn, and J. Bachmann, RCS Adv. 7, 37627 (2017).

[30] J. Kimling, F. Kronast, S. Martens, T. Böhnert, M. Martens, J. Herrero-Albillos, L. Tati-Bismaths, U. Merkt, K. Nielsch, and G. Meier, Phys. Rev. B 84, 174406 (2011).

[31] S. Jamet, S. Da Col, N. Rougemaille, A. Wartelle, A. Locatelli, T. O. Menteş, B. Santos Burgos, R. Afid, L.
Cagnon, S. Bochmann, J. Bachmann, O. Fruchart, and J. C. Toussaint, Phys. Rev. B 92, 144428 (2015).

[32] http://feellgood.neel.cnrs.fr.

[33] See Supplemental Material at http://link.aps.org/ supplemental/10.1103/PhysRevLett.123.217201 for additional details on sample fabrication, MFM and XMCD PEEM imaging, pulse width and DW velocity and error calculations, Joule heating, micromagnetic simulations, and DW inertia simulations, which includes Refs. [34-43].

[34] S. Kadowaki and M. Takahashi, J. Phys. Soc. Jpn. 50, 1154 (1981).

[35] K. Ikeda, Trans. Jpn. Inst. Met. 29, 183 (1988).

[36] T. Nishizawa and K. Ishida, Bull. Alloy Phase Diagrams 4, 390 (1983).

[37] B. G. Tóth, L. Péter, A. Révész, J. Pádár, and I. Bakonyi, Europhys. J. B 75, 167 (2010).

[38] L. Aballe, M. Foerster, E. Pellegrin, J. Nicolas, and S. Ferrer, J. Synchrotron Radiat. 22, 745 (2015).

[39] M. Foerster, J. Prat, V. Massana, N. Gonzalez, A. Fontsere, B. Molas, O. Matilla, E. Pellegrin, and L. Aballe, Ultramicroscopy 171, 63 (2016).

[40] F. Warkusz, J. Phys. D 11, 689 (1978).

[41] M. N. Ou, S. R. Harutyunyan, S. J. Lai, C. D. Chen, T. J. Yang, and Y. Y. Chen, Phys. Status Solidi (b) 244, 4512 (2007).

[42] A. Thiaville, Y. Nakatani, F. Piéchon, J. Miltat, and T. Ono, Europhys. J. B 60, 15 (2007).

[43] K. Ueda, T. Koyama, R. Hiramatsu, D. Chiba, S. Fukami, H. Tanigawa, T. Suzuki, N. Ohshima, N. Ishiwata, Y. Nakatani, K. Kobayashi, and T. Ono, Appl. Phys. Lett. 100, 202407 (2012).

[44] S. Da Col, S. Jamet, M. Staňo, B. Trapp, S. L. Denmat, L. Cagnon, J. C. Toussaint, and O. Fruchart, Appl. Phys. Lett. 109, 062406 (2016).

[45] O. Boulle, G. Malinowski, and M. Kläui, Mater. Sci. Eng. R 72, 159 (2011).

[46] T. Gushi, M. J. Klug, J. P. Garcia, H. Okuno, J. Vogel, J. Attané, T. Suemasu, S. Pizzini, and L. Vila, https://arxiv.org/ abs/1901.06868.

[47] M. Sturma, C. Bellegarde, J.-C. Toussaint, and D. Gusakova, Phys. Rev. B 94, 104405 (2016).

[48] D. Aurelio, A. Giordano, L. Torres, G. Finocchio, and E. Martinez, IEEE Trans. Magn. 49, 3211 (2013).

[49] V. Uhlir, S. Pizzini, N. Rougemaille, V. Cros, E. Jimenez, L. Ranno, O. Fruchart, M. Urbanek, G. Gaudin, J. Camarero, C. Tieg, F. Sirotti, E. Wagner, and J. Vogel, Phys. Rev. B 83, 020406(R) (2011).

[50] R. Hertel, J. Phys. Condens. Matter 28, 483002 (2016). 